УДК 621.833 .681

\title{
ВОЛНОВОЙ ТОРЦЕВОЙ КИНЕМАТИЧЕСКИЙ РЕДУКТОР ДЛЯ ПОРШНЕВЫХ НАСОСОВ ПРИ ДОБЫЧЕ ТЯЖЕЛОЙ НЕФТИ
}

\author{
Джасем Мохамад Али',2, \\ jasemma@tpu.ru
}

\author{
Крауиньш Петр Янович 1 , \\ peterkrau@tpu.ru \\ 1 Национальный исследовательский Томский политехнический университет, \\ Россия, 634050, г. Томск, пр. Ленина, 30. \\ 2 Университет Аль-Фурат, \\ Сирия, г. Дейр-эз-Зор, ул. Университетская, 7.
}

\begin{abstract}
Актуальность работы обусловлена необходимостью обеспечения работоспособности редукторов для поршневых насосов в суровых условиях эксплуатации при добычи высоковязкой нефрти из малодебитных нефтяных скважин. Возможность повышения нагрузочной способности обычного эвольвентного зацепления за счет его рациональной геометрии, применения качественных материалов и термообработки не безграничны. Поэтому проблема создания новых видов механических передач и систем зацеплений является особо актуальной.

Цель: определить зависимость продолжительности сопряжения зубьев от рабочего профиля зуба волнового торцевого кинематического редуктора за один цикл прецессии $\varphi$.

Объекты: волновой торцевой кинематический редуктор как составная часть поршневых скважинных насосов в суровых условиях эксплуатации для добычи высоковязкой нефтии из малодебитных скважин.

Методы: методы матричной и векторной алгебры, численные методы решения систем нелинейных уравнений, теория механизмов и машин и основная теория пространственных передач зацеплением.

Результаты. Рассмотрена проблема создания волнового торцевого кинематического редуктора с многопарным зацеплением. Синтез многопарного зацепления для волнового ториевого кинематического редуктора, обеспечивающего постоянство передаточной функции, предусматривает: разработку математической модели зацепления с учетом особенностей взаимодействия зубьев при пространственно-сферическом движении; описание профиля зубьев системой уравнений на сферическую поверхность и на нормальное сечение зубьев для внутреннего зацепления; выявление с помощью программы MathCAD 2010 Professional математического эксперимента и определение области существования 100\%-го многопарного сопряжения зубьев путем сравнения формы их профиля с характером аналитической функции многопарного зацепления.
\end{abstract}

\section{Ключевые слова:}

Нефоть, волновой торцевой кинематический редуктор, поршневой насос, передаточное отношение, многопарное зацепление, длина рабочего профиля.

\section{Введение}

Добыча тяжелой нефти - развивающаяся технология добычи тяжелой нефти в промышленных количествах. Расчетные резервы тяжелой нефти - более 6 трлн баррелей, в три раза больше обычных нефти и газа. Факторы, которые влияют на трудность вклада запасов в производство, включают проницаемость, пористость, глубину и давление. Плотность и вязкость нефти - основные факторы, которые определяют способ добычи тяжелой нефти $[1,2]$.

Центробежные насосы ограничено применяются в работе с высоковязкими жидкостями из-за фрикционных потерь внутри насоса, которые вызывают снижение скорости потока, поэтому при добыче высоковязкой нефти применяют поршневые насосы [3].

Одной из самых важных частей насосного оборудования является редуктор из-за его прямого воздействия на улучшение энергетических и массогабаритных показателей насосных агрегатов [4].

Анализ современных тенденций совершенствования систем зацеплений, числовой характеристики и требований потребителей к техническому уровню выпускаемых редукторов показал целесообразность исследования и разработки волновых редукторов с новым видом многопарного зацепления [4, 5 и др.].

Многопарность зацепления обеспечивает повышение несущей способности редуктора в целом, улучшает энергетические и массогабаритные показатели, повышает кинематическую точность, снижает уровень напряжений в контакте и улучшает геометрические и гидродинамические условия смазки взаимодействующих зубьев и др. Поэтому применение волновых редукторов с многопарным зацеплением в силовых приводах машин является весьма эффективным [6].

Высокая кинематическая точность волновых редукторов с выпукло-вогнутым профилем зубьев, их компактность и широкий диапазон передаточных функций, а также возможность полного устранения зазоров в зацеплении и даже создание некоторого преднатяга делают выгодным их применение в механизмах, используемых в добывающей промышленности, исполнительных приводах промышленных роботов и манипуляторов, в металлорежущих станках, и других механизмах, требующих высокой точности позиционирования рабочих органов $[7,8]$.

Специфика пространственно-сферического движения шестерни волнового торцевого кинематиче- 
ского редуктора позволяет решать одну из основных проблем герметизации - передавать движение в абсолютно герметизированное пространство через сплошную непроницаемую стенку, что открывает широкие перспективы его применения в вакуумной технике, нефтехимической и атомной промышленности, космической и авиационной областях $[9,10]$.

Рассматривается кинематика взаимодействия зубьев и особенности преобразования движения, излагаются требования и условия существования многопарного зацепления, даются рекомендации по выбору профиля зубьев и его параметров $[11,12]$. Это позволит конструкторам правильно подобрать параметры зацепления в зависимости от конкретного назначения проектируемого редуктора [13].

На рис. 1 представлена принципиальная схема волнового торцевого кинематического редуктора, состоящего из пяти элементов: входного вала (генератора волн) - 1, выполненного в виде кривошипного валика, блока шестерен - 3, состоящей из двух конических колес внутреннего зацепления, которые свободно вращаются на входном вале, неподвижного конического колеса - 2 с наружным зацеплением и подвижного конического колеса - 4 , жестко связанного с выходным валом - 5. Левый зубчатый конический венец $\left(z_{2}\right)$ блока шестерен -3 находится в зацеплении с неподвижным коническим колесом - 2, а правый зубчатый конический венец $\left(z_{3}\right)$ - с подвижным коническим колесом - 4 [14].

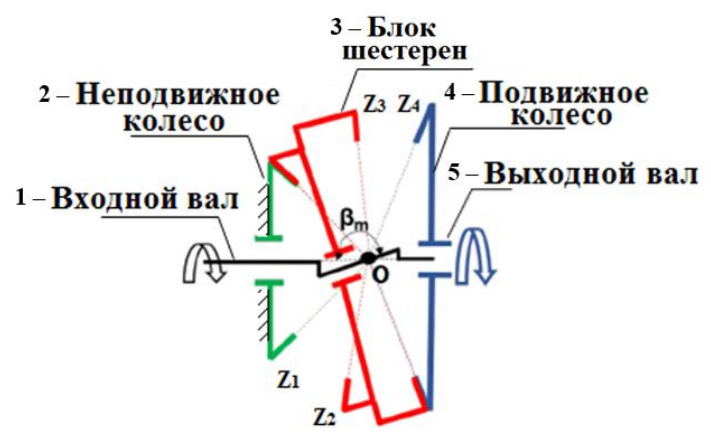

Pис. 1. Структурная схема волнового ториевого кинематического редуктора

Fig. 1. Structural scheme of the wave face kinematic reducer

Количество зубьев неподвижного колеса и зубьев сопряженного с ним блока шестерен равны $\left(z_{1}=z_{2} \pm 1\right)$, а число зубьев $\left(z_{3}\right)$ блока шестерен - 3 и подвижного колеса - $4\left(z_{4}\right)$ совпадает. В рассматриваемом редукторе делительные конусы неподвижного колеса - 2, блока шестерен -3 и подвижного колеса -4 имеют общую вершину, расположенную в точке О и лежащую на оси редуктора [15].

При вращении входного вала - 1 блок шестерен - 3 совершает сферическое движение относительно точки $O$ посредством обкатывания зубьями венца $z_{2}$ зубья $z_{1}$ неподвижного колеса - 2; одновременно входят в зацепление и зубья венца $z_{3}$ с зубьями колеса $z_{4}$. Благодаря этому блок шестерен - 3 передает вращение зубчатому колесу -4 , жестко соединенному с выходным валом - 5 [16].
Как известно $[17,18]$, тело, которое совершает сферическое движение, имеет три степени свободы. Следовательно, положение такого тела может быть определено тремя параметрами относительно неподвижной системы координат $O X Y Z$. В теоретической механике [18], как правило, положение тела, совершающего сферическое движение, определяют при помощи углов Эйлера. Для этого свяжем жестко систему координат $O X_{1} Y_{1} Z_{1}$ с шестерней (рис. 2).

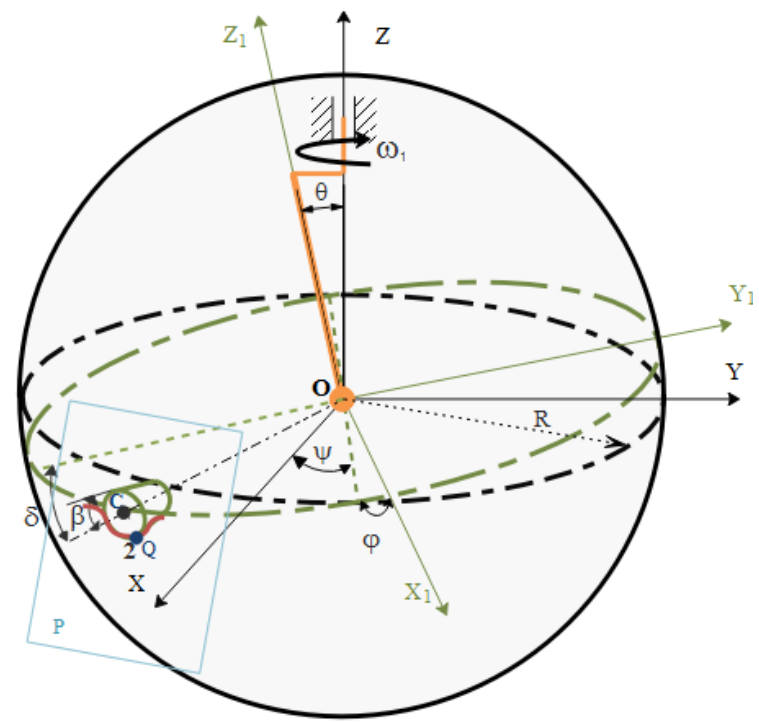

Pис. 2. Описание сферического движения иестерни волнового ториевого кинематического редуктора

Fig. 2. Description of the spherical movement of the gear of the wave face kinematic reducer

Применительно к волновому торцевому кинематическому редуктору угол $\psi$ - это угол прецессии (угол поворота ведущего вала), угол $\theta-$ угол нутации (угол наклона кривошипного участка ведущего вала к оси ведомого вала), $\varphi$ - угол собственного вращения шестерни вокруг собственной геометрической оси.

Отношение между углом прецессии $\psi$ и углом собственного вращения $\varphi$ определяется формулой:

$$
\varphi=-\frac{Z_{1}}{Z_{2}} \psi,
$$

где $Z_{1}, Z_{2}$ - числа зубьев сопряженных колес.

Точка $C$ движется по сферической поверхности радиуса $R$ с центром прецессии в точке $O$ (рис. 2). Зная траекторию движения центра зуба, находим положение точки его контакта с зубом колеса, совокупность семейства которых за цикл прецессии представляет собой профиль зубьев этого колеса. Положение точки С определяется координатами (1)

$$
X_{C}=R \cos \delta\left[\left(\begin{array}{l}
-\cos \psi\left(\frac{Z_{1}}{Z_{2}} \psi\right)+ \\
\left.+\sin \psi \cos \left(\frac{Z_{1}}{Z_{2}} \psi\right) \cos \theta\right)
\end{array}\right)\right]-
$$$$
-R \sin \delta \sin \psi \sin \theta \text {; }
$$ 


$$
\left.Y_{C}=-R \cos \delta\left[\begin{array}{l}
\left(\sin \psi\left(\frac{Z_{1}}{Z_{2}} \psi\right)+\right. \\
+\cos \psi \cos \left(\frac{Z_{1}}{Z_{2}} \psi\right) \cos \theta
\end{array}\right)\right]-
$$

$-R \sin \delta \cos \psi \sin \theta$;

$$
Z_{C}=-R \cos \delta \cos \left(\frac{Z_{1}}{Z_{2}} \psi\right) \sin \theta-R \sin \delta \cos \theta .
$$

Определяем координаты точки контакта зубьев $Q$, совокупность которых представляет собой профиль зубьев входного колеса на сфере по формулам (2)-(4).

$$
\begin{gathered}
X_{Q}=\frac{R^{2} \cos \beta-\left(k_{1} Z_{Q}-d_{Q}\right) Y_{C}-Z_{Q} Z_{C}}{X_{C}}= \\
=\frac{\left(k_{1} Y_{C}+Z_{C}\right)}{X_{C}} Z_{Q}+\frac{\left(R^{2} \cos \beta+d_{1} Y_{C}\right)}{X_{C}} \Rightarrow \\
X_{Q}=k_{2} Z_{Q}-d_{2},
\end{gathered}
$$

где введены обозначения

$$
\begin{gathered}
k_{2}=\frac{\left(k_{1} Y_{C}+Z_{C}\right)}{X_{C}} ; d_{2}=\frac{\left(R^{2} \cos \beta+d_{1} Y_{C}\right)}{X_{C}} . \\
Y_{Q}=\frac{\left\lfloor X_{C}\left(X_{C} \cdot v_{C_{X}}+Y_{C} \cdot v_{C_{Y}}\right)+Z_{C}{ }^{2} \cdot v_{C_{X}}\right\rfloor_{Z}}{\left(X_{C} \cdot v_{C_{Y}}-Y_{C} \cdot v_{C_{X}}\right) Z_{C}} Z_{Q} \\
-\frac{R^{2} \cos \beta \cdot v_{C_{X}}}{\left(X_{C} \cdot v_{C_{Y}}-Y_{C} \cdot v_{C_{X}}\right)} \Rightarrow Y_{Q}=k_{1} Z_{Q}-d_{1},
\end{gathered}
$$

где введены обозначения

$$
\begin{gathered}
k_{1}=\frac{\left\lfloor X_{C}\left(X_{C} \cdot v_{C_{X}}+Y_{C} \cdot v_{C_{Y}}\right)+Z_{C}{ }^{2} \cdot v_{C_{X}}\right\rfloor}{\left(X_{C} \cdot v_{C_{Y}}-Y_{C} \cdot v_{C_{X}}\right) Z_{C}} \\
d_{1}=\frac{R^{2} \cos \beta \cdot v_{C_{X}}}{\left(X_{C} \cdot v_{C_{Y}}-Y_{C} \cdot v_{C_{X}}\right)} \cdot \\
Z_{Q}=\frac{\left(k_{1} d_{1}-k_{2} d_{2}\right)}{k_{1}^{2}+k_{2}^{2}+1}- \\
-\frac{\sqrt{\left(k_{1} d_{1}-k_{2} d_{2}\right)^{2}+\left(k_{1}^{2}+k_{2}^{2}+1\right)\left(R^{2}-d_{1}^{2}-d_{2}^{2}\right)}}{k_{1}^{2}+k_{2}^{2}+1}
\end{gathered}
$$

Чтобы представить профиль зубьев колес в нормальном сечении, мы спроектируем со сферы на плоскость $P_{1}$, перпендикулярную зубу (рис. 2).

Для этого сначала определяем координаты точек $Q_{1}$ и $Q_{2}$ (рис. 2), соответствующие двум последовательными минимумам кривой профиля на сфере.

Выберем в плоскости $P_{1}$ систему координат $Q_{1} \tau \lambda$, ось $Q_{1} \tau$ которой проходит через точку $Q_{2}$.

Проекцию профиля зубьев со сферы на плоскости $P_{1}$ находим путем определения координат точек $Q_{N}$ пересечения с этой плоскостью семейства прямых, проходящих через центр прецессии $O$, и соответствующих точек профиля зубьев на сфере.

И после ряда преобразований получаем проекцию на плоскость профиля зубьев колеса, определяемую декартовыми координатами, через которые перейдем от координат $X_{N}, Y_{N}, Z_{N}$ к координатам $\tau, \lambda$ :

$$
\begin{gathered}
\tau=\frac{\left[\left(Q_{1} Q_{2}\right)^{2}+\left(Q_{1} Q_{N}\right)^{2}-\left(Q_{2} Q_{N}\right)^{2}\right]}{2\left(Q_{1} Q_{1}\right)} ; \\
\lambda=\sqrt{\left(Q_{1} Q_{N}\right)^{2}-\tau^{2}} .
\end{gathered}
$$

Теперь находим параметрические уравнения кривой, которую описывает ось зуба шестерни на плоскости $P_{1}$, т. е. переход от координат $X_{G}, Y_{G}, Z_{G}$ к координатам $\tau_{1}, \lambda_{1}$ осуществляем согласно формулам (6)

$$
\begin{gathered}
\tau_{1}=\frac{\left[\left(Q_{1} Q_{2}\right)^{2}+\left(Q_{1} Q_{G}\right)^{2}-\left(Q_{2} Q_{G}\right)^{2}\right]}{2\left(Q_{1} Q_{1}\right)} ; \\
\lambda_{1}=\sqrt{\left(Q_{1} Q_{G}\right)^{2}-\tau_{1}^{2}} .
\end{gathered}
$$

Зависимость $\lambda_{1}$ от $\tau_{1}$ представляет собой траекторию движения центра зуба шестерни на плоскости $P_{1}$.

Зависимость $\lambda=f(\tau)$ представляет собой огибающую кривую множества дуг окружностей радиусом $R$, центры которых расположены на траектории движения точки $C$, описываемой уравнением $\lambda_{1}=f\left(\tau_{1}\right)$.

Результаты экспериментов. Графическое построение функций $\lambda=f(\tau)$ и $\lambda_{1}=f\left(\tau_{1}\right)$ осуществлялось в совмещенных координатах $\lambda_{1} \lambda-\tau_{1} \tau$ при значениях угла прецессии $0 \leq \psi \leq 2 \pi$, т. е. при одном обороте ведущего вала. При этих значениях $\psi$ уравнениями (5) описывается профиль одного зуба колеса.

В программе MathCAD 2010 Professional был создан широкий спектр модифицированных профилей зубов с различными геометрическими параметрами (рис. 3, $а, б$ ).

О многопарности зацепления можно судить по графикам функций $\lambda_{1}=f\left(\tau_{1}\right)$ и $\lambda=f(\tau)$ (рис. $\left.3, a\right)$, представляющих соответственно проекцию траектории движения центра $C$ зуба шестерни и профиля зуба колеса на плоскости $P_{1}$ (рис. 2). Каждому значению функции $\lambda_{1}=f\left(\tau_{1}\right)$ соответствует определенная точка на графике функции $\lambda=f(\tau)$, являющаяся точкой контакта взаимодействующих зубьев. Положение любой точки контакта на графике $\lambda=f(\tau)$ определяется углом прецессии $\psi$.

Исходные условия получения функции $\lambda=f(\tau)$ обеспечивают следующее взаимодействие зубьев: зуб шестерни с центром, расположенным в точке $C$, контактирует с профилем зуба колеса в точке $Q$.

При этом множество окружностей (рис. 3, a) с центрами, которые расположены на участках

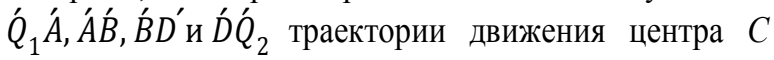
зуба, огибается кривыми $Q_{1} A, A B, B D$ и $D Q_{2}$. Видно, что кривы $Q_{1} A$ и $Q_{2} D$ пересекаются в очке $Q_{N}$. Это доказывает, что взаимодействие зубьев блока шестерен и колеса по ломаной кривой $Q_{1} Q_{N} Q_{2}$ представляет собой профиль зуба колеса. На участке $Q_{N} A B D Q_{N}$ взаимодействие зубьев отсутствует [19]. По размерам фигуры $Q_{N} A B D Q_{N}$ можно судить о многопарности зацепления, так как продолжительность взаимодействия зубьев определяет многопарность зацепления, т. 
е. размер фигуры $Q_{N} A B D Q_{N}-$ это периметр, который характеризует продолжительность отсутствия контакта. Чем больше размеры этой фигуры, тем меньше многопарность зацепления, и на оборот. При отсут- ствии этой фигуры многопарность зацепления составляет $\varepsilon=100 \%$, так как зубья взаимодействуют между собой непрерывно (рис. 3, б).

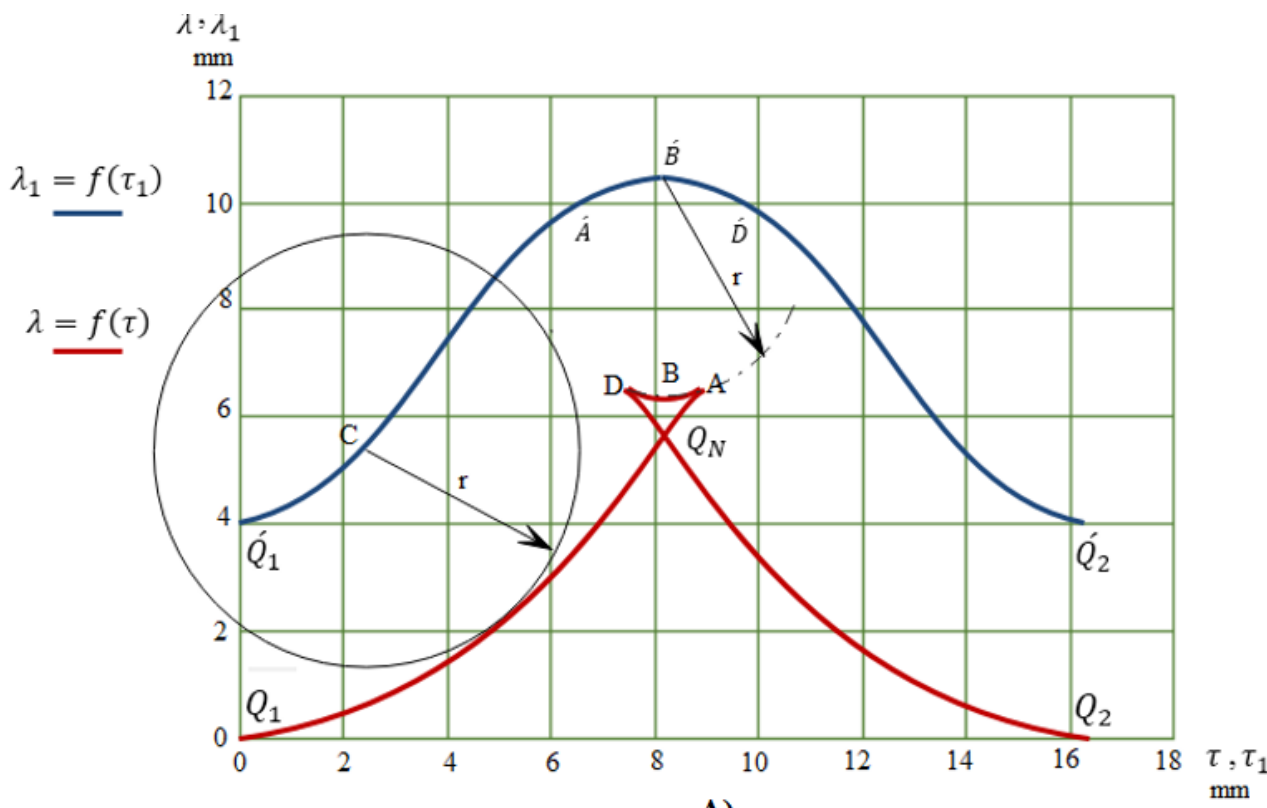

A)

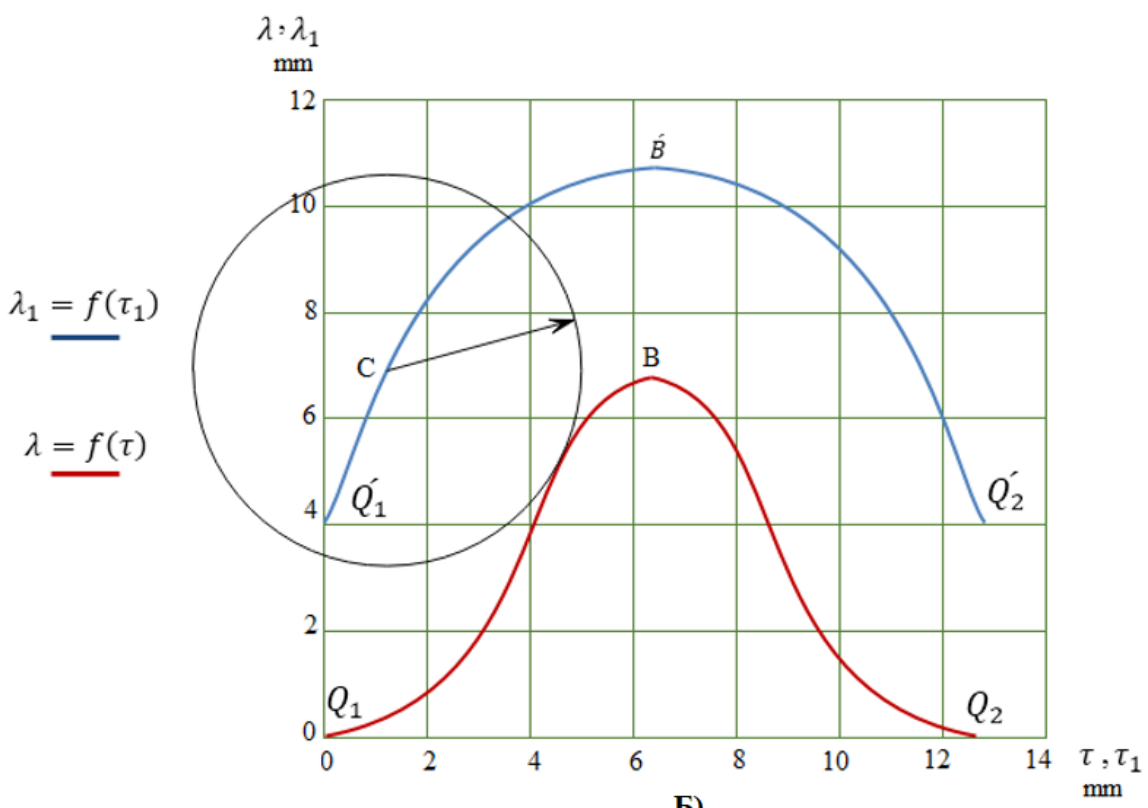

Pис. 3. Взаимодействие зубьев за один прецессионный иикл при многопарности зацеплении при: $A$ ) $Z_{I}=29, Z_{2}=30$, $D=150 \mathrm{~mm}, r=4 \mathrm{~mm}, \theta=2,5^{\circ}, \delta=0^{\circ}, \varepsilon<100 \%$; Б) $Z_{I}=29, Z_{2}=30, D=150 \mathrm{~mm}, r=4 \mathrm{~mm}, \theta=2,5^{\circ}, \delta=30^{\circ}, \varepsilon<100 \%$

Fig. 3. Teeth interaction for one precession cycle at a multiple-tooth contact at: A) $Z_{1}=29, Z_{2}=30, D=150 \mathrm{~mm}, \mathrm{r}=4 \mathrm{~mm}$, $\theta=2,5^{\circ}, \delta=0^{\circ}, \varepsilon<100 \%$; Б) $Z_{1}=29, Z_{2}=30, D=150 \mathrm{~mm}, r=4 \mathrm{~mm}, \theta=2,5^{\circ}, \delta=30^{\circ}, \varepsilon<100 \%$

Многопарность зацепления волнового торцевого кинематического редуктора определяется продолжительностью сопряжения зубьев за один цикл прецессии $0<\varphi<\frac{Z_{1} \psi}{Z_{2}}[20,21]$. Из рис. $3, a$ видно, что продолжительность сопряжения зубьев зависит от формы их рабочего профиля [22].

Для определения рабочего профиля зуба $Q_{1} Q_{n}$ (рис. 3) на MathCAD 2010 Professional исследована зависимость $\tau=\tau(\varphi)$ в интервале $0<\varphi<\frac{Z_{1} \psi}{Z_{2}}$, т. е. за один цикл.

Поскольку продолжительность контакта зубьев, а следовательно, и многопарность зацепления зависят от формы профиля зубьев [23], для исследования были приняты функции $\tau=\tau(\varphi)$ характерных профилей: a) с наличием у вершины фигуры $Q_{N} A B D Q_{N}$ заострения (рис. $3, A$ ), б) с закругленной вершиной (рис. 3,5 ). 

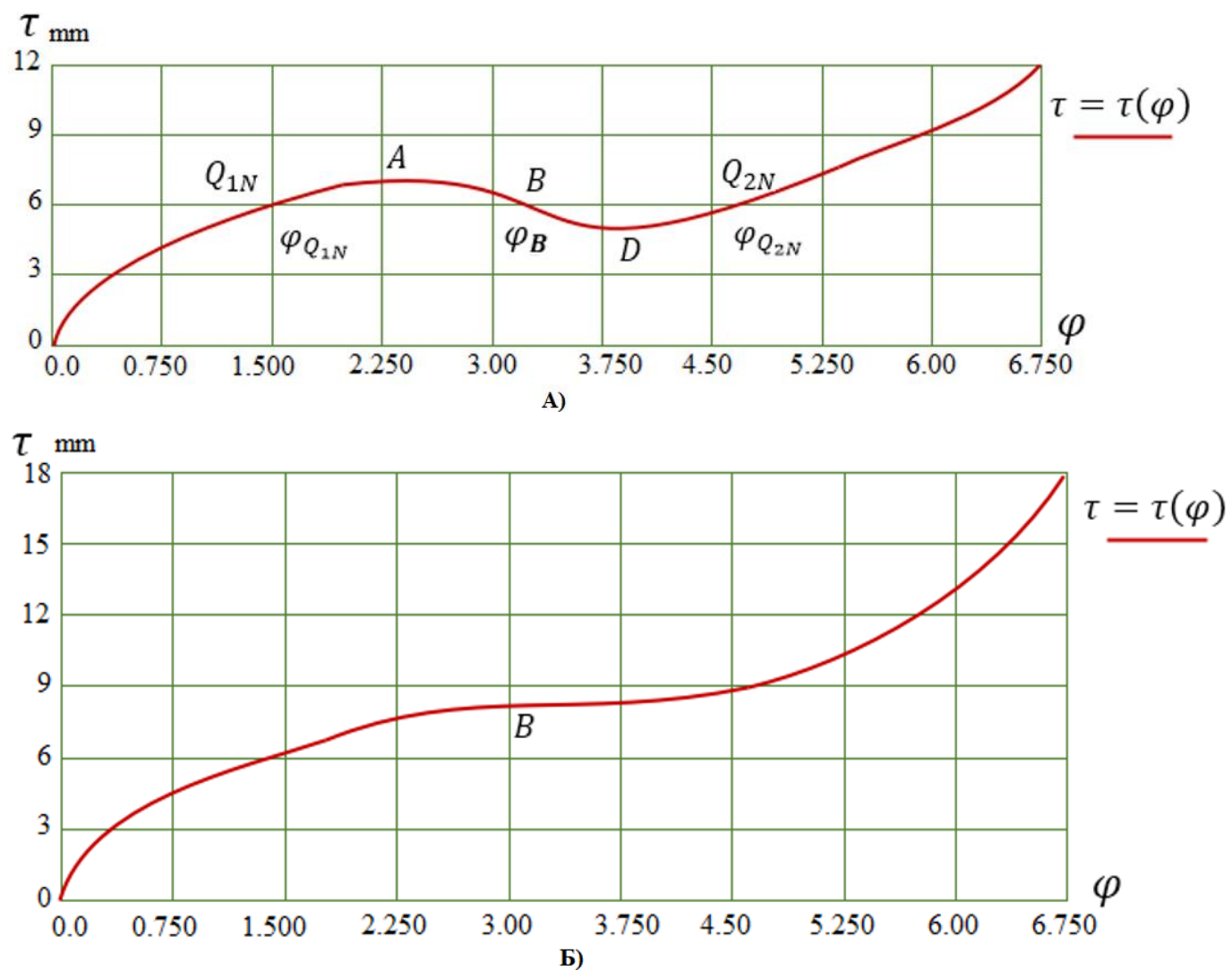

$\tau_{\mathrm{mm}}$

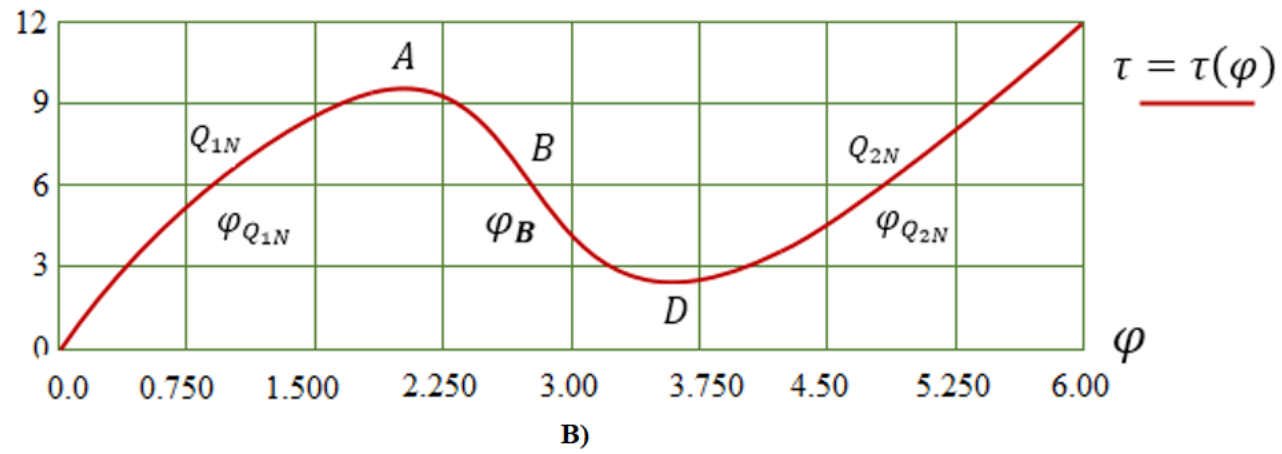

$\tau \mathrm{mm}$

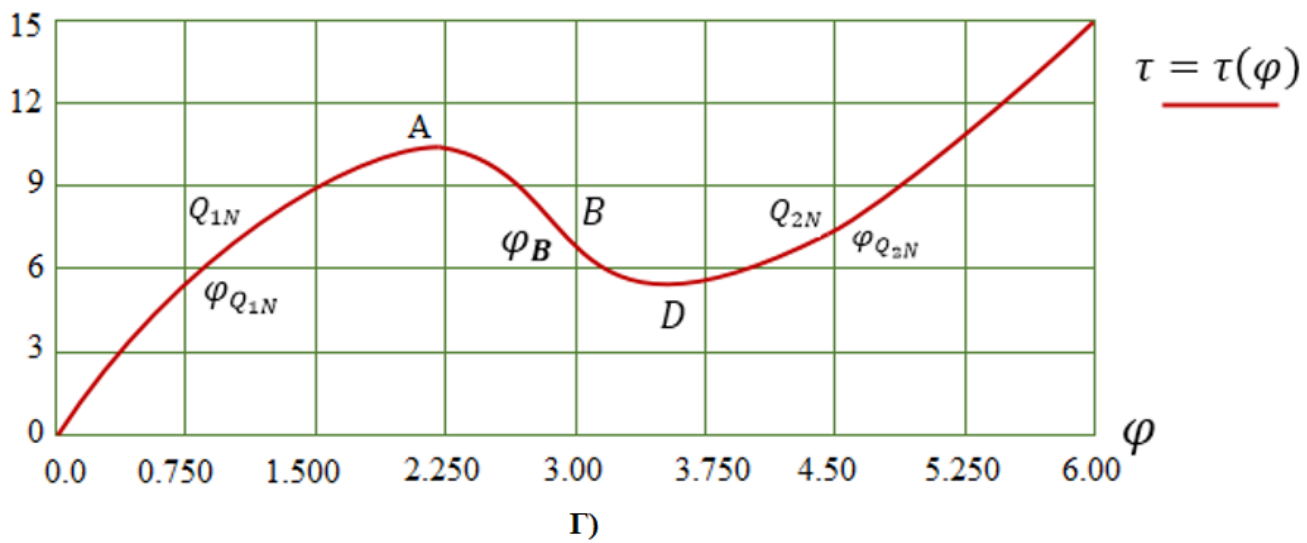

Pис. 4. Определение многопарности зацепления при: A) $Z_{I}=29, Z_{2}=30, D=150 \mathrm{~mm}, r=4 \mathrm{~mm}, \theta=2,5^{\circ}, \delta=0^{\circ}$; Б) $Z_{I}=29$, $Z_{2}=30, D=150 \mathrm{~mm}, r=4 \mathrm{~mm}, \theta=2,5^{\circ}, \delta=30^{\circ}$; B) $Z_{I}=29, Z_{2}=30, D=150 \mathrm{~mm}, r=4 \mathrm{~mm}, \theta=2,5^{\circ}, \delta=22,5^{\circ}$; $\left.\Gamma\right) Z_{1}=22$, $Z_{2}=21, D=150 \mathrm{~mm}, r=4 \mathrm{~mm}, \theta=2,5^{\circ}, \delta=22,5^{\circ}$

Fig. 4. Definition of multiple-tooth contact at: A) $Z_{l}=29, Z_{2}=30, D=150 \mathrm{~mm}, r=4 \mathrm{~mm}, \theta=2,5^{\circ}, \delta=0^{\circ}$; Б) $Z_{1}=29, Z_{2}=30$, $D=150 \mathrm{~mm}, r=4 \mathrm{~mm}, \theta=2,5^{\circ}, \delta=30^{\circ}$; $\left.\left.B\right) Z_{l}=29, Z_{2}=30, D=150 \mathrm{~mm}, r=4 \mathrm{~mm}, \theta=2,5^{\circ}, \delta=22,5^{\circ} ; \Gamma\right) Z_{l}=22, Z_{2}=21$, $D=150 \mathrm{~mm}, r=4 \mathrm{~mm}, \theta=2,5^{\circ}, \delta=22,5^{\circ}$ 
Анализ функции $\tau=\tau(\varphi)$ показывает также, что уравнение

$$
\tau\left[\left(\frac{Z_{2}}{Z_{1}}\right) \psi-\tau\left(\frac{Z_{2}}{Z_{1}}\right) \pi\right]=0,
$$

определяющее продолжительность рабочего профиля, имеет три корня:

$$
\varphi_{Q_{1 N}}, \varphi=\left(\frac{z_{2}}{Z_{1}}\right) \pi \text { и } \varphi_{Q_{2 N}} .
$$

Рабочий профиль зубьев существует на участке $Q Q_{n}$ графика функции $\tau=\tau(\varphi)$, т. е. при $0<\varphi<\varphi_{Q_{1 N}}$ определяется из условия

$$
\tau(\varphi)-\tau\left[\left(\frac{z_{2}}{z_{1}}\right) \pi\right]<0 .
$$

Исходя из вышеизложенного, многопарность зацепления (в \%) определяется по формуле

$$
\varepsilon=\frac{\varphi_{Q_{1 N}}}{\varphi_{B}} 100 \%,
$$

где $\varphi_{Q_{1 N}}$ - наименьший корень уравнения (7).

По формулам (7)-(9) была составлена зависимость $\tau=\tau(\varphi)$ в программе MathCAD 2010 Professional за один цикл.

Из рис. $4, a$, , , 2 видно, что функция $\tau=\tau(\varphi)$ иммет два экстремума - A и $\mathrm{D}$, соответствующие точкам A и $\mathrm{D}$ функции $\lambda=f(\tau)$, а функция на рис. $4, \sigma$ непрерывно

\section{СПИСОК ЛИТЕРАТУРЬ}

1. Speight J.G. Heavy oil recovery and upgrading. - Oxford: Gulf Professional Publishing, 2019. $-839 \mathrm{p}$

2. Simanzhenkov V., Raphael I. Crude oil chemistry. - Florida: $\mathrm{Crc}$ Press, 2003. -402 p.

3. Corvaglia A., Massimo R. Comparison of $0 \mathrm{D}$ and 3D hydraulic models for axial piston pumps // Energy procedia. - 2018. V. 148. - P. 114-121.

4. Litvin F.L., Fuentes A. Gear geometry and applied theory. 2nd ed. New York: Cambridge University Press, 2004. -800 p.

5. Vullo V. Gears. V. 1: Geometric and Kinematic Design. - Cham: Springer International Publishing A\&G, 2020. - V. 1. - 844 p.

6. Litvin F.L., Fuentes A., Howkins M. Design, generation and TCA of new type of asymmetric face-gear drive with modified geometry // Computer methods in applied mechanics and engineering. - 2001. - V. 190 (43-44). - P. 5837-5865.

7. Cervantes Sánchez J.J., Rico Martínez J.M., Panduro Calvario C. A general and systematic framework for the kinematic analysis of complex gear systems // Meccanica. - 2012. - V. 47. - № 1. - P. 3-21.

8. Batsch M. Mathematical model and tooth contact analysis of convexo-concave helical bevel Novikov gear mesh // Mechanism and Machine Theory. - 2020. - V. 149. - P. 103842.1-103842.18.

9. Lin C., He C., Hu Y. Analysis on the kinematical characteristics of compound motion curve-face gear pair // Mechanism and Machine Theory. - 2018. - V. 128. - P. 298-313.

10. Saribay Z.B., Bill R.C. Design analysis of Pericyclic Mechanical Transmission system // Mechanism and Machine Theory. 2013. - V. 61. - P. 102-122.

11. Mathematical modeling and simulation of the external and internal double circular-arc spiral bevel gears for the nutation drive / L. Yao, B. Gu, S. Haung, G. Wei, J.S. Dai // Journal of Mechanical Design. 2010. - V. 132 (2). - P. 021008.1-021008.10.

12. Tooth contact analysis with latent error of double circular-arc spiral bevel gears for industrial robot joint nutation drive / Z. Lin, L. Yao, J. Zhang, T. Su, K. Chen // Journal of the Brazilian Society of Mechanical Sciences and Engineering. - 2020. - V. 42 (1). P. 1-12.

13. Influence analysis of system parameters on characteristics of the nutation drive with double circular arc spiral bevel gears / Y. Cai, L. Yao, Z. Xie, J. Zhang, C. Peng // Forschung Im Ingenieurwesen. - 2017. - V. 81 (2). - P. 125-133. растет. Непрерывный рост функции $\tau=\tau(\varphi)$ свидетельствует о том, что многопарность зацепления $\varepsilon=100 \%$, a наличие экстремумов - 0 прерывистом контакта зубьев, т. е. многопарность $\varepsilon<100 \%$.

\section{Заключение}

Таким образом, разработан волновой торцевой кинематический редуктор с многопарным зацеплением для поршневых насосов, пользующихся при добыче высоковязкой нефти. Чтобы обеспечить многопарное зацепление, необходимо учитывать влияние каждого из параметров $-\delta, \theta$ и $Z$ - на форму рабочего профиля зуба. При изменении одного из этих параметров рабочий профиль зуба меняется, следовательно, меняется продолжительность сопряжения зубьев за один цикл прецессии $[9,24]$.

Установлено, что продолжительность взаимодействия зубьев определяет многопарность зацепления, о которой можно судить по размерам фигуры $Q_{N} A B D Q_{N}$, периметр которой характеризует продолжительность отсутствия контакта $[19,25]$. Чем больше размеры этой фигуры, тем меньше многопарность зацепления, и на оборот. При отсутствии этой фигуры многопарность зацепления составляет $\varepsilon=100 \%$, т. е. зубья взаимодействуют между собой непрерывно.

14. Jasem M., Krauinsh P.Y. Analysis of the Stress-strain State (SSS) under static load of nutation speed reducer // Journal of Advanced Research in Dynamical and Control Systems. - 2019. - V. 11. № 5-SI. - P. 137-141.

15. Jasem M.A., Krauinsh P.Y. Kinematical analysis of the nutation speed reducer // IOP Conference Series: Materials Science and Engineering. IOP Publishing. - 2019. - P. 012022.

16. Джасем М.А., Крауиньш П.Я. Математическая модель зацепления и аналитическое описание профиля зубьев волнового торцевого кинематического редуктора // Омский научный вестник. - 2021. - № 1 (175). - С. 22-31.

17. Айрапетов Э.Л. Совершенствование методов расчета нагруженности и прочности передач зацеплением // Техника машиностроения. - 2001. - № 2. - С. 8-34.

18. Журавлев В.Ф. Основы теоретической механики. 2-е изд. - М.: Физматлит, 2001. - 320 с

19. Шевелева Г.И., Волков А.Э., Медведев В.И. Программное обеспечение производства конических и гипоидных зубчатых передач с круговыми зубьями // Техника машиностроения. 2001. - № 2 (30). - С. 40-51.

20. Гольдфарб В.И., Исакова Н.В. Варианты спироидных передач с точки зрения реализации винтового параметра // Передачи и трансмиссии. - 1995. - № 1. - С. 25-34.

21. Лагутин С.А. Синтез пространственных зацеплений методом винтов // Передачи и трансмиссии. - 1999. - № 2. - С. 59-70.

22. Колесников В.К., Котликова В.Я. О технологическом синтезе пространственных зубчатых передач с локализованным контактом // Проблемы машиностроения и надежности машин. 1993. - № 1. - С. 86-91.

23. Журавлев Г.А. Оценка применимости решения Герца в задачах о контакте зубьев колес // Техника машиностроения. 2001. - № 2. - С. 82-90.

24. Волков А.Э., Шевелева Г.И. Компьютерный анализ работы конических и гипоидных зубчатых передач // Проблемы машиностроения и надежности машин. - 2001. - № 5. - С. 96-103.

25. Мазуру С. Оценка точности обработки зубьев зубчатых колес с нестандартным профилем // Сборник трудов XV MHTK «Машиностроение и техносфера XXI века». - Севастополь: Изд-во ЧП «Технополис», 2008. - Т. 2. -326 с.

Поступила 19.01.2022 г. 


\section{Информация об авторах}

Джасем М.А., аспирант отделения материаловедения Инженерной школы новых производственных технологий Национального исследовательского Томского политехнического университета; ассистент кафедры механического проектирования и производства Факультета машиностроения и электротехники Университета АльФурат.

Крауиньш П.Я., доктор технических наук, профессор отделения материаловедения Инженерной школы новых производственных технологий Национального исследовательского Томского политехнического университета. 
UDC 621.833 .681

\title{
WAVE FACE KINEMATIC REDUCER FOR PISTON PUMPS DURING EXTRACTION OF HEAVY OIL
}

\author{
Mohamad A. Jasem 1,2 , \\ jasemma@tpu.ru
}

Peter Ya. Kraunish', peterkrau@tpu.ru

1 National Research Tomsk Polytechnic University, 30, Lenin avenue,Tomsk, 634050, Russia.

2 Al-Furat University,

7, University street, Deir ez-Zor, Syria.

The relevance of the research is caused by the need of ensuring the efficiency of gear reducers for piston pumps in the harsh operating conditions for high-viscosity oil extraction from low production wells. The possibility of increasing the load capacity of the usual involute engagement due to its rational geometry, the use of high-quality materials and heat treatment is not limitless. Therefore, the problem of creating new types of mechanical gears and engagement systems is particularly relevant.

The main aim of the research is to determine the dependence of the duration of mating teeth on the working tooth profile of the wave face kinematic reducer for one cycle of precession $\varphi$.

Objects: the wave face kinematic reducer as a part of piston pumps in the harsh operating conditions during the extraction of highviscosity oil from low production wells.

Methods: methods of matrix and vector algebra, numerical techniques of the nonlinear equations, theory of mechanisms and machines, the main theory of spatial gear.

Results. The paper considers the problem of creating a wave face kinematical reducer with multiple-tooth contact. The synthesis of multipair gearing for a wave face kinematical reducer, which ensures the constancy of transfer function, provides for: development of a mathematical model of gearing taking into account the peculiarities of the interaction of teeth during special-spherical motion; description of the profile of the teeth by a system of equations for a spherical surface and for a normal section of teeth for internal gearing; identification via MathCAD 2010 Professional program of a mathematical experiment and determination of the area of existence of $100 \%$ multiple-tooth contact of teeth by comparing the shape of their profile with the nature of the analytical function of multiple-tooth contact.

\section{Key words:}

Crude oil, wave face kinematic reducer, piston pump, gear ratio, multiple-tooth contact, working profile length.

\section{REFERENCES}

1. Speight J.G. Heavy oil recovery and upgrading. Oxford, Gulf Professional Publishing, 2019. 839 p.

2. Simanzhenkov V., Raphael I. Crude oil chemistry. Florida, $\mathrm{Crc}$ Press, 2003. 402 p.

3. Corvaglia A., Massimo R. Comparison of $O D$ and $3 D$ hydraulic models for axial piston pumps. Energy procedia, 2018, vol. 148, pp. 114-121.

4. Litvin F.L., Fuentes A. Gear geometry and applied theory. $2^{\text {nd }}$ ed. New York, Cambridge University Press, 2004. 800 p.

5. Vullo V. Gears: Vol. 1: Geometric and Kinematic Design. Cham, Springer International Publishing A\&G, 2020. vol. 1.844 p.

6. Litvin F.L., Fuentes A., Howkins M. Design, generation and TCA of new type of asymmetric face-gear drive with modified geometry. Computer methods in applied mechanics and engineering, 2001, vol. 190 (43-44), pp. 5837-5865.

7. Cervantes Sánchez J.J., Rico Martínez J.M., Panduro Calvario C. A general and systematic framework for the kinematic analysis of complex gear systems. Meccanica, 2012, vol. 47, no. 1, pp. 3-21.

8. Batsch M. Mathematical model and tooth contact analysis of convexo-concave helical bevel Novikov gear mesh. Mechanism and Machine Theory, 2020, vol. 149:103842, pp. 103842.1103842.18 .

9. Lin C., He C., Hu Y. Analysis on the kinematical characteristics of compound motion curve-face gear pair. Mechanism and Machine Theory, 2018, vol. 128, pp. 298-313.

10. Saribay Z.B., Bill R.C. Design analysis of Pericyclic Mechanical Transmission system. Mechanism and Machine Theory, 2013, vol. 61, pp. 102-122.

11. Yao L., Gu B., Haung S., Wei G., Dai J.S. Mathematical modeling and simulation of the external and internal double circular-arc spiral bevel gears for the nutation drive. Journal of Mechanical Design, 2010, vol. 132 (2), pp. 021008.1 - 021008.10.
12. Lin Z., Yao L., Zhang J., Su T., Chen K. Tooth contact analysis with latent error of double circular-arc spiral bevel gears for industrial robot joint nutation drive. Journal of the Brazilian Society of Mechanical Sciences and Engineering, 2020, vol. 42 (1), pp. 1-12.

13. Cai Y., Yao L., Xie Z., Zhang J., Peng C. Influence analysis of system parameters on characteristics of the nutation drive with double circular arc spiral bevel gears. Forschung $I m$ Ingenieurwesen, 2017, vol. 81 (2), pp. 125-133.

14. Jasem M., Krauinsh P.Y. Analysis of the Stress-strain State (SSS) under static load of nutation speed reducer. Journal of Advanced Research in Dynamical and Control Systems, 2019, vol. 11, no. 5SI, pp. 137-141.

15. Jasem M.A., Krauinsh P.Y. Kinematical analysis of the nutation speed reducer. IOP Conference Series: Materials Science and Engineering. IOP Publishing, 2019, $012022 \mathrm{p}$.

16. Jasem M.A., Krausinsh P.Ya. Matematicheskaya model zatsepleniya i analiticheskoe opisanie profilya zubev volnovogo tortsevogo kinematicheskogo reduktora [Mathematical model of the engagement and analytical description of the tooth profile of the wave face kinematic reducer]. Omskiy nauchnyi vestnik, 2021, no. 1 (175), pp. 22-31.

17. Ayrapetov E.L. Improving methods for calculating loading and gearing strength. Q: Engineering Engineering, 2001, no. 2, pp. 8-34.

18. Zhuravlev V. Osnovy teoreticheskoy mekhaniki [The auxiliary bases of theoretical mechanics]. $2^{\text {nd }}$ ed. Moscow, Fizmatlit Publ., 2001. $320 \mathrm{p}$

19. Shevelev G.I., Volkov A.E., Medvedev V.I. Programmnoe obespechenie proizvodstva konicheskikh i gipoydnykh zubchatykh peredach s krugovymi zubyami [Software manufacturing conical and hypoid gears with circular teeth]. Tekhnika mashinostroeniya, 2001, no. 2 (30), pp. 40-51.

20. Goldfarb V.I., Isakova N.V. Varianty spiroydnykh peredach s tochki zreniya realizatsii vintovogo parametra [Spiroid 
transmissions from the point of view of the screw parameter] Peredachi i transmissii, 1995, no. 1, pp. 25-34.

21. Lagutin S.A. Sintez prostranstvennykh zatsepleniy metodom vintov [Synthesis of spatial clutches by screws]. Peredachi $i$ transmissii, 1999, no. 2, pp. 59-70.

22. Kolesnikov V.K, Kotlikova V.Ya. O tekhnologicheskom sinteze prostranstvennykh zubchatykh peredach s lokalizovannym kontaktom [About the technological synthesis of spatial gears with localized contact]. Problemy mashinostroeniya $i$ nadezhnosti mashin, 1993, no. 1, pp. 86-91.

23. Zhuravlev G.A. Otsenka primenimosti resheniya Gertsa v zadachakh o kontakte zubev koles [Assessment of the applicability of the Hertz solution in the tasks of contact of the teeth of the wheels]. Tekhnika mashinostroeniya, 2001, no. 2, pp. 82-90.
24. Volkov A.E., Sheveleva G.I. Kompyuterny analiz raboty konicheskikh i gipoydnykh zubchatykh peredach [Computer analysis of the work of conical and hypoid gears]. Problemy mashinostroeniya i nadezhnosti mashin, 2001, no. 5, pp. 96-103.

25. Mazuru C. Otsenka tochnosti obrabotki zubev zubchatykh koles s nestandartnym profilem [Evaluation of the precision processing of teeth gears with non-standard profile]. Sbornik trudov XV MNTK. Mashinostroenie i tekhnosfera XXI veka [Collection of works of the XV MNTK. Mechanical Engineering and Technosphere of the XXI century]. Sevastopol, PE «Technopolis» Publ., 2008. Vol. 2, $326 \mathrm{p}$

Received: 19 January 2022.

\section{Information about the authors}

Mohamad A. Jasem, postgraduate student, National Research Tomsk Polytechnic University; assistant, Al-Furat University, Deir ez-Zor.

Peter Ya. Kraunish, Dr. Sc., professor, National Research Tomsk Polytechnic University. 